Luciano Carlos Rezzoagli* Martin Cammarata"

Universidad Nacional del Litoral, Santa Fe, Argentina

Consejo Nacional de Investigaciones Científicas y Técnicas (Conicet), Argentina

Recibido: 15 de diciembre de 2015 Concepto de evaluación: 28 de abril de 2016

Aprobado: 20 de octubre de 2016

Artículo de investigación

(C) 2017 Universidad Católica de Colombia. Facultad de Ciencias

Económicas y Administrativas. Todos los derechos reservados

* Abogado, Magíster y Doctor en Finanzas y Derecho Tributario, Especialista en Bloques Económicos, en Sistema Financiero Mundial, en Aspectos Regulatorios del Comercio Exterior y en Política

Económica Internacional. Investigador adjunto del Conicet y la Universidad Nacional del Litoral. Profesor titular de Derecho Financiero, Tributario y Aduanero en la Facultad de Ciencias Jurídicas

y Sociales de la Universidad Nacional del Litoral, Santa Fe, Argentina. Director del Grupo de Investigación de Federalismo Fiscal y Asimetrías Regionales en la República Argentina, de la misma Universidad. Dirección de correspondencia: Centro de Investigación, Secretaría de Ciencia y Técnica, Facultad de Ciencias Jurídicas y Sociales (UNL), Cándido Pujato 2751 (C P. 3000), ciudad de Santa Fe, provincia de Santa Fe, Argentina. Correo electrónico: Irezzoagli@hotmail.com o Irezzoagli@fcjs.unl.edu.ar

\section{(Re)discutir el federalismo fiscal como instrumento de desarrollo social en la República Argentina*}

\section{RESUMEN}

Transcurridos más de veinte años desde la reforma constitucional argentina, sin que se haya dado cumplimiento a la cláusula transitoria sexta, que establecía la realización de una nueva ley de coparticipación federal, y pese a los importantes logros en la disminución de la pobreza, el rompimiento de la pobreza estructural y la consolidación de la clase media desde 2001 hasta 2015, importantes desequilibrios se mantienen en Argentina respecto a la igualdad de oportunidades entre los ciudadanos por región individualmente considerada, lo cual constituye un desafío y una prioridad de agenda para el Estado. El federalismo fiscal, como fenómeno e instrumento en pos del desarrollo social, debe atender a la igualación de oportunidades como objetivo central, en función del cual se podrá repensar toda la estructura vertical de competencias, capacidades y responsabilidades gubernamentales. Un desafío importante es dar mayor implicancia al proceso bottom-up en la configuración o reconfiguración de políticas públicas, actualmente signadas por la discrecionalidad y el direccionamiento, para buscar políticas consensuadas y cercanas a los ciudadanos en todos los rincones del país.

Palabras clave: federalismo fiscal, igualdad de oportunidades, asimetrías regionales, políticas públicas, sistema tributario, sistema financiero.

JEL: H53, O23.

\section{(Re)discuss the fiscal federalism as a tool of social development in Argentian Republic}

\section{ABSTRACT}

After more than 20 years since the constitutional reform, not having complied with the sixth provisional clause, and despite the progress made in reducing poverty and strengthening the middle class since 2001 to date,

\footnotetext{
* El presente artículo científico es financiado y desarrollado en el marco del proyecto "(Re)pensando el federalismo fiscal en la República Argentina: un enfoque jurídicoinstitucional", del Consejo Nacional de Investigaciones Científicas y Técnicas (Conicet) y la Universidad Nacional del Litoral.
} 
** Abogado, estudiante del Doctorado en Derecho de la Universidad Nacional del Litoral (UNL), Santa Fe, Argentina. Investigador del Grupo $\mathrm{CAI}+\mathrm{D}$ denominado Federalismo Fiscal y Asimetrías Regionales en la República Argentina, Facultad de Ciencias Jurídicas y Sociales (FCJS) de la misma universidad. Dirección de correspondencia: Centro de Investigación, Secretaría de Ciencia y Técnica, Facultad de Ciencias Jurídicas y Sociales (UNL), Cándido Pujato 2751 (C P. 3000), ciudad de Santa Fe, provincia de Santa Fe, Argentina. Correo electrónico: martincamma_07@hotmail.com significant imbalances remain in Argentina with regard to the equality of opportunities among citizens from different regions of the country, which is both a challenge and a priority in the State's agenda. Fiscal Federalism as a phenomenon and instrument for social development must attend to the equalization of opportunities as a central objective, according to which it will be possible to rethink the entire vertical structure of governmental competencies, capacities and responsibilities. A major challenge is to give greater impetus to the bottom-up process in the configuration or reconfiguration of public policies, currently marked by discretion and direction, to seek consensus policies and close to citizens in all corners of the country.

Keywords: fiscal federalism, equality of opportunities, public policies, regionals asymmetries, taxation system, financial system.

\section{(Re)discutir o federalismo fiscal como instrumento de desenvolvimento social na República Argentina}

\section{RESUMO}

Após mais de 20 anos da reforma constitucional argentina, sem que tenha sido cumprida a cláusula transitória sexta, que estabelecia a realização de uma nova lei de coparticipação federal, e, embora os importantes progressos na diminuição da pobreza, o rompimento da pobreza estrutural e a consolidação da classe média desde 2001 até 2015, importantes desiquilíbrios se mantêm na Argentina quanto à desigualdade de oportunidades entre os cidadãos por região individualmente considerada, o que constitui um desafio e uma prioridade de agenda para o Estado. $\mathrm{O}$ federalismo fiscal, como fenômeno e instrumento em prol do desenvolvimento social, deve atender à igualação de oportunidades como objetivo central, em função do qual poderá ser repensada toda a estrutura vertical de competências, capacidades e responsabilidades governamentais. Um desafio importante é dar maior implicância ao processo bottom-up na configuração ou reconfiguração de políticas públicas, atualmente marcadas pela discricionariedade e pelo direcionamento para buscar políticas consensuais e próximas dos cidadãos em todos os lugares do país.

Palavras-chave: assimetrias regionais, federalismo fiscal, igualdade de oportunidades, políticas públicas, sistema financeiro, sistema tributário. 


\section{INTRODUCCIÓN}

El objetivo central de este trabajo científico es discutir y repensar el rol del sistema tributario como instrumento y del federalismo fiscal como fenómeno, tomando como punto inicial el reconocimiento y la aceptación de un gran margen de asimetrías regionales en la República Argentina, desde una visión revisionista, solidaria y con justicia social. La metodología empleada es de tinte cualitativa, deductiva y descriptiva-analítica, y toma como fuente empírica los datos actuales que publica el Instituto Nacional de Estadísticas y Censos (Indec), el último Censo Nacional de Hogares del 2010, los datos que publica la Comisión Económica para América Latina y el Caribe (Cepal), el Banco Mundial y Naciones Unidas, y la doctrina especializada en federalismo fiscal tanto en el ámbito nacional como internacional. De manera conjunta se tiene en cuenta la normatividad histórica y actual (constitucional y legal) existente en la República Argentina en esta área, con las limitantes que implica la precisión en la estructura y el desarrollo de un artículo científico.

En función de las apreciaciones vertidas, es de suma relevancia, en forma cronológica, conocer y entender el principio de igualdad de oportunidades y sus implicancias y desafíos en América Latina, especialmente en Argentina. Ello implica examinar los desequilibrios regionales a través de indicadores ejemplificativos relevantes (de elección no programática) que vislumbran los datos actuales publicados por el Indec, la Cepal, el Banco Mundial, Naciones Unidas y trabajos científicos empíricos de doctrina especializada. Estos desafíos se analizan en relación con la pertinencia normativa (las máximas constitucionales respecto al federalismo fiscal, la solidaridad y la búsqueda de un desarrollo igualitario en todos los rincones de la nación, en atención a la elaboración de políticas públicas diferenciadas) ${ }^{2}$ y el actual sistema

2 Generados a partir de la reforma de 1994, los artículos 16, 37 (segundo párrafo) y 75 (inciso 2, párrafo 3, e incisos 19 y 23) destacan dentro de su terminología y en forma relacional un concepto particular de igualdad: la de oportunidades. Tal uso del lenguaje opera como un reconocimiento implícito operativo del federalismo fiscal como fenómeno y la coparticipación federal como instrumento.

\section{LA DESIGUALDAD DE OPORTUNIDADES EN ARGENTINA, ELEMENTO CLAVE PARA REPLANTEAR EL FEDERALISMO FISCAL COMO INSTRUMENTO DE DESARROLLO ${ }^{3}$}

La búsqueda del desarrollo social dirigido a la inclusión, la justicia social y la igualdad requiere, por lo tanto, fomentar no solo la integración sino también la erradicación de la pobreza. Un país es socialmente justo cuando todas las personas que lo integran poseen los mismos derechos y tienen, potencialmente, sin condicionantes exógenos limitantes, las mismas posibilidades de desarrollar sus capacidades sin limitaciones exógenas, así como el derecho de acceder, en cantidad y calidad, a las prestaciones que brinda el Estado, como los servicios públicos indispensables.

Así, para alcanzar una vida digna, el desarrollo humano debe estar asegurado; entonces, la igualdad, desde una visión social, refiere a un mínimo común de derechos y obligaciones que corresponden a la totalidad de los integrantes de la sociedad (Barbieri, 2007). En vista de este objetivo, se necesita una actividad estatal tendiente a garantizar el cumplimiento de los derechos individuales y colectivos, así como brindar los medios idóneos para fomentar las potencialidades de las personas — son, principalmente, las físicas, cognitivas, emocionales, relacionales e intelectuales-.

de la realidad dispar dentro del país. Por esto, las máximas constitucionales aparecen vinculadas con acciones positivas, como lo es la educación gratuita o la (re)distribución de la recaudación impositiva. Dicho en otros términos, la equidad manda a justipreciar la especificidad de la diferencia, en el marco de una política igualitaria (Barbieri, 2007).

3 La Organización de Naciones Unidas (ONU, 2015) define desarrollo como el aprovechamiento igualitario de las condiciones sociales y materiales de los habitantes de un pueblos y su paulatino mejoramiento, bajo el marco del respeto de sus valores culturales. En este sentido, los vínculos críticos entre la pobreza, la desigualdad económica, el déficit de trabajo decente o digno y la exclusión social han sido reconocidos como los obstáculos sustanciales para el cumplimiento del desarrollo, y así está plasmado en la Cumbre Mundial sobre Desarrollo Social, realizada en Copenhague en 1995. 
En otras palabras, la igualdad de oportunidades es una forma de concebir la justicia social.

El Informe sobre Desarrollo Humano para América Latina y El Caribe, presentado en 2010 por el Programa de Naciones Unidas para el Desarrollo (PNUD), es contundente al identificar a América Latina, al igual que en informes anteriores, como el continente más desigual del mundo; por ello, existe una necesidad prioritaria de profundizar en el diagrama, la instrumentalización y la consolidación de políticas redistributivas.

La distinción entre elementos o circunstancias exógenas al individuo (no dependientes) - tales como el origen social, el lugar de nacimiento, el género o el acceso a educación- de aquellos que los individuos adquieren por acciones dependientes a lo largo de su ciclo de vida — por ejemplo, el esfuerzo en el trabajo y en los estudios- resulta fundamental para comprender este principio de igualdad en su matriz de desarrollo horizontal de oportunidades. Aunque esta distinción es por cierto discutible en la práctica, las dimensiones por igualar entre individuos debieran abarcar solo aquellas que son exógenas - por ejemplo, garantizar por acciones positivas el acceso a la educación-, y se debe respetar, aunque impulsando su disminución, la diferencia que provenga de la propia agencia de las personas.

Este reconocimiento e identificación de condicionantes exógenos resulta importante para diagramar políticas públicas que brinden un marco adecuado para las oportunidades de desarrollo de cada individuo en cualquier punto del país. En la medida en que exista acceso desproporcionado de algún sector poblacional a la concreción de sus derechos humanos, relacionado con factores externos o independientes a las posibilidades personales - como el género, el lugar de origen, su color de piel, su estatus económico, etc.-, claramente habrá causas constitutivas de la desigualdad de oportunidades. Así, el principio de igualdad, entendido desde su faceta material de brindar y garantizar las mismas oportunidades, es aquel que pregona la eliminación de obstáculos exógenos que impiden o restringen a las personas para lograr, por sus propios medios (ámbito endógeno), una vida digna ${ }^{4}$.

En una sociedad como la actual, todas las diferencias económicas que encuentran sus causas en elementos exógenos se traducen claramente en desigualdades sociales, pues no hay poder político capaz de impedir que aquellas diferencias se traduzcan en relaciones de dependencia, sometimiento o dominación. Aunque existan proveedores materiales de bienestar y protección - como las familias, las organizaciones sociales y comunitarias y, de manera más amplia, el propio mercado-, corresponde al Estado garantizar y hacer cumplir los derechos sociales, culturales y económicos de los habitantes, el acceso a estos, así como instrumentalizar, controlar y evaluar la regulación del mercado de trabajo para fomentar el empleo formal y digno (Cecchini y Vargas, 2014).

Generalmente, las políticas públicas están alineadas a intereses particulares de ciertos actores nacionales o regionales (Rezzoagli y Gamberg, 2015), aun cuando sean presentadas bajo un solemne marco de bien común y formalicen la instrumentalización de objetivos nacionales o regionales. Estos actores que alinean las políticas públicas a sus intereses personales tergiversan el sentido y el contenido de la política que se propone inclusiva y democrática (Fernández, Seval y Vigil, 2013).

Una dificultad por señalar es que con frecuencia el acceso a estas oportunidades de desarrollo, en términos individuales y colectivos, se encuentra condicionado por diversos factores que obstaculizan el ingreso de los sectores más vulnerables de la sociedad, o de permitirlo, lo hacen en grados mínimos y con diferencia en términos de duración y calidad. Así, para ejemplificar el análisis vertido, si consideramos el acceso de familias ricas y pobres a la energía eléctrica en su casa, puede asegurarse que las familias relativamente ricas tendrán electricidad casi en cualquier país del mundo;

$4 \quad$ Las construcciones argumentativas no nacen desde el vacío intelectual; para esta conceptualización se han seguido las ideas respecto a las "capacidades básicas" de Sen (2000), "alisar el camino" de Roemer (1998), "oportunidades para el bienestar" de Anderson (1999) y "acceso a las ventajas" de Cohen (1991). 
pero en países como Panamá, Perú y Nicaragua, entre otros muchos que podrían mencionarse en el ámbito continental, menos del $20 \%$ de las familias pobres la tienen (PNUD, 2010).

Lo mismo puede decirse, entre otras consideraciones, respecto al acceso a cualquier servicio público (Rezzoagli y Bazza, 2012) o frente a la restricción de las posibilidades de desarrollo laboral o educativo (Rezzoagli y Gamberg, 2014). Así, puede aseverarse que resulta poco probable que los niños más capaces en términos académicos, pero carentes de recursos económicos adecuados, obtengan las mejores oportunidades educativas en función de dichas capacidades; por el contrario, es mucho más probable que la pobreza relativa restrinja sus posibilidades educativas, fundamentalmente en comparación con niños de entornos más pudientes en términos económicos, que adquieren un acceso desproporcionado a las mejores escuelas. En función de esto, resulta indispensable analizar la actividad estatal tendiente a nivelar lo que la sociedad y el mercado no asumen de manera espontánea.

Esta diferencia entre los ciudadanos dentro de la escala geográfica que quiere considerarse (local, provincial, nacional o internacional) es lo que marca la desigualdad de oportunidades. De sostenerse en el tiempo esta desigual situación, las consecuencias negativas serán alarmantes en virtud del acaparamiento de oportunidades. Este es el proceso que tiene lugar cuando grupos concretos controlan tanto los recursos como los activos más preciados para su propio interés, con lo cual se perpetuán y acrecientan las desigualdades y los ciclos de dominación y dependencia (Rezzoagli y Gamberg, 2014).

En su informe De la estabilización a un crecimiento sostenido, el Fondo Monetario Internacional (2014) manifiesta que la desigualdad ya no constituye exclusivamente un tema de justicia social, sino que también se enmarca como un obstáculo del crecimiento económico de los países. Esta es una novedad que cierra una brecha histórica en el pensamiento económico oficial. En este sentido, siempre que la sociedad y el Estado tengan como objetivos la justicia social, la consecución de la libertad y el progreso económico, será la igualdad, necesariamente, un pilar y una bandera elemental e imprescindible (Oszlak, 2007). Asimismo, es conveniente destacar que ya el mismo concepto de nación debe entenderse como arco de solidaridades que exceden los diversos y contrapuestos intereses de la sociedad civil.

Ahora bien, el desarrollo de capacidades, el acceso a prestaciones y a redes de contención social y el incremento de oportunidades laborales a lo largo y ancho del tejido social pueden encontrarse con barreras que impidan su difusión igualitaria. Aunque resulte paradójico, analizar y comprender el grado de bienestar de distintos grupos de una sociedad conduce rápidamente a asociarlo con sus opuestos más significativos (Velázquez, 2010); por eso, amén de la diversidad de factores que condicionan la realidad de los distintos sectores sociales, se considerarán a continuación, tanto individual como conjuntamente, solo algunos que por su grado de importancia e impacto social los elevan como indicadores relevantes. El motivo por el cual se seleccionaron estos factores por sobre otros que son igualmente determinantes responde exclusivamente a una cuestión de delimitación de información representativa de lo que se pretende abordar en este artículo.

Repasando la historia argentina, podemos obtener datos significativos y relevantes (Cao y Vaca, 2006); por ello, y con ánimo de no ser repetitivos con trabajos anteriores, se resume lo más relevante en la siguiente cita:

Durante el siglo XIX la instauración de la Aduana Única en el Puerto de Buenos Aires y el trazado de las vías del ferrocarril convergentes al mismo favorecieron la concentración de población y actividades como también la centralización de funciones en esta región. Dicha concentración poblacional se acentuó durante la vigencia del modelo de Industrialización por Sustitución de Importaciones (ISI) debido a que las principales industrias se radicaron en los centros urbanos de 
la zona pampeana, incentivando las migraciones internas hacia esa región. El emprendimiento agroexportador de base pampeana ocupó el centro de la dinámica nacional, generándose una brecha de desarrollo con el resto de los territorios. Como resultante de ese proceso histórico, aunado a otras variables no analizadas, las zonas periféricas perdieron importancia demográfica (actualmente concentran menos del 35\% de la población total) y productiva (su producto bruto geográfico es inferior al $20 \%$ del total). De esa manera, la República Argentina terminó respondiendo a los moldes clásicos de una profunda aglomeración poblacional y productiva en grandes centros urbanos. [...] Por su parte, también vale la pena destacar que las cuatro jurisdicciones argentinas más importantes (C.A.B.A., provincia de Buenos Aires, provincia de Santa Fe y provincia de Córdoba) contienen en su territorio al $62,52 \%$ de los habitantes de la Argentina, a pesar de que su superficie apenas representa el $21 \%$ del total. Un estudio de más de 25 clasificaciones de provincias realizadas por distintos investigadores muestra que esta visión tiene un amplio consenso entre los que se dedican al tema (Cao, Rubins y Vaca, 2003).

La Cepal (2013), al presentar su trabajo Lineamientos estratégicos para el desarrollo industrial en Argentina establece, en palabras de su secretaria ejecutiva Alicia Bárcena:

Desde el año 2003 la República Argentina ha transitado por un sendero de crecimiento, progreso técnico, creación de empleo y reducción de la pobreza sin precedentes en más de medio siglo, lo cual logró revertir en gran medida el daño económico y social producido por la dinámica neoliberal de los noventa. (p. 17)
Se puede cuantificar la pobreza mediante la elección y utilización de cualquiera de estos tres métodos internacionalmente reconocidos: a) la línea de pobreza $(\mathrm{LP})^{5}$; b) las necesidades básicas insatisfechas (NBI) ${ }^{6}$, utilizado por la Cepal en función de los datos de la Encuesta Permanente de Hogares de 2010, y c) el método integrado, que es el más completo, pero a la fecha registra escasa utilización.

Velázquez, Mikkelsen, Linares y Celemín (2014), al analizar la calidad de vida en Argentina, ranking del bienestar por departamentos en 2010, establecen:

Los progresos han sido insoslayables en la mayoría de las dimensiones de la calidad de vida, hay un sustancial incremento en educación, una disminución a la mitad de la población con bajo nivel de instrucción e incremento al doble de los graduados universitarios durante la década 20002010, también existen importantes logros en salud, como la disminución a la mitad de la mortalidad infantil y la sensible reducción de la población sin cobertura social. Datos alentadores que muestran

5 Este método utiliza el ingreso o el gasto de consumo como medida para diferenciar a las personas pobres; para ello, otorga un valor per cápita de una canasta mínima de consumo necesario para la sobrevivencia, y según sea el ingreso o el gasto, en función de dicha canasta se puede determinar el estatus. La línea de pobreza extrema corresponde al valor per cápita de la canasta que contiene los rubros alimenticios exclusivamente, en tanto la línea de pobreza total comprende el valor per cápita de los rubros totales, y no exclusivamente los alimenticios. En el Informe de pobreza y desigualdad por ingresos en la Argentina Urbana 20102015, el Observatorio de Deuda Social Argentina (2016) utiliza este método y manifiesta que la tasa de pobreza en el país fue del $29 \%$ a fines de 2015 , y la de indigencia fue del 5,3\% en el mismo periodo. La crítica más relevante en la que coinciden varios especialistas respecto de este informe es la de una posible sobrevaloración de la inflación con que se mide la canasta básica total (CBT), al valorizar bienes y servicios diversos exclusivamente con la inflación en alimentos, que necesariamente siempre es la más elevada. 6 Este segundo indicador para medir cuantitativamente la pobreza se basa en necesidades básicas estructurales como el acceso a la salud, a los servicios públicos, a la vivienda, a la educación, entre otros. Este indicador permite visualizar a la población en estatus de pobreza cuando tengan una necesidad básica insatisfecha, y a pobres extremos cuando tengan insatisfechas dos o más necesidades. 
que el país ha podido, aunque sea en estos aspectos, superar la nefasta etapa de los noventa y su estallido económico y social en el año 2001. (p. 161)

Por su parte, Narodowski y Panigo (2010) afirman que los resultados respecto al crecimiento conjunto del empleo y el poder adquisitivo de los trabajadores ha generado una reducción de los indicadores de pobreza, indigencia y desigualdad frente a la situación que presentaba el país en 2001; resultados oportunamente intensificados por la puesta en marcha de distintas políticas sociales, entre las cuales se destaca la articulación y puesta en marcha de la Asignación Universal por Hijo.

Indudablemente, dicho estallido del 2001 generó un cambio de paradigma ideológico en la República Argentina, con repercusiones económicas y de política discursiva. Así, se observa un posicionamiento gubernamental tendiente a disminuir la pobreza como política estratégica, un mejoramiento de salarios de empleados en relación de dependencia y de ingresos de autónomos mediante el aprovechamiento de condiciones de competitividad de commodities en el comercio exterior, como también la implementación de programas sociales que atendieron necesidades vitales de los ciudadanos con desiguales efectos de aprovechamiento (Narodowski y Panigo, 2010).

Desde el comienzo de la década kirschnerista, empezó a articularse con mayor intensidad un conjunto de programas de Transferencias Monetarias Condicionadas (en adelante, TMC) destinados a grupos vulnerables. Entre estos se resaltan el Programa Manos a la Obra, el de Familia y el de Seguros de Capacitación y Empleo. Dichos programas de TMC fueron una profundización y continuación del Plan Trabajar (1996). Se los denomina programas de TMC porque las personas beneficiadas debían cumplir ciertos requisitos exigidos por cada uno de los programas como condición sine qua non para recibir el monto en efectivo; por ello, recibieron la denominación de corresponsabilidad.
Durante el transcurso de 2009 surgen y se consolidan en el ámbito nacional dos programas de TMC, bajo dependencia directa del Ministerio de Desarrollo Social de la Nación y la Administración Nacional de la Seguridad Social (Anses): Argentina Trabaja y Asignación Universal por Hijo para la Protección Social (AUH).

El Programa Ingreso Social con Trabajo, Argentina Trabaja, que surge a mediados de 2009, tuvo entre sus principales ejes de intervención la inclusión social a través del trabajo, la capacitación desde una perspectiva integral y la promoción de la organización cooperativa. Sus principales beneficiarios son aquellas personas pertenecientes a hogares en situación de alta vulnerabilidad social que no cuenten con otro ingreso proveniente de trabajo registrado, pensión, jubilación, programa social nacional o provincial incompatible.

La implementación del programa se lleva adelante a través de una articulación directa con los distintos entes ejecutores en el ámbito municipal, representados por las distintas secretarías que realizan diversas obras públicas, y donde los beneficiarios del programa se encuentran organizados bajo la modalidad de cooperativas de hasta 60 miembros. Esta distribución apunta al cumplimiento de uno de los componentes pilares del programa. Desarrollar una actividad laboral concreta en una sede municipal es condición para poder ser beneficiario de la transferencia directa de dinero que asciende a una suma de $\$ 1200$ (U\$ 150) y es depositada mensualmente en una cuenta individual y personal en calidad de monotributista social. Desde agosto de 2012, y tras cumplirse tres años del lanzamiento del programa, al ingreso mensual que reciben los beneficiarios se sumaron dos nuevos conceptos que pueden percibir: un plus por productividad de $\$ 300$ (U\$35) y otro por presentismo de $\$ 250$ (U\$30).

A su vez, la intervención del programa se organiza a través de otra serie de componentes educativos y sanitarios. En el marco de su participación en el programa, los beneficiarios pueden optar por acceder a algún tipo de terminalidad educativa (alfabetización, educación primaria 
o secundaria), capacitarse en algún oficio o formación laboral específica y participar en jornadas de prevención y promoción de la salud. Esta serie de componentes configuran los criterios de admisión y condicionalidad monetaria establecidos por el programa.

La Asignación Universal Por Hijo para la Protección Social (AUH) y la Asignación Universal por Hijo con Discapacidad, creadas bajo Decreto P. E. N. 1602/9 en octubre de 2009, y cuya implementación efectiva corresponde al primer trimestre de 2010, fue concebida con el objetivo de equiparar el ingreso de aquellos niños de sectores más vulnerables, beneficiando así directamente a los hijos de las personas que no estuvieran incorporados al mercado de trabajo formal y, por consiguiente, no recibieran la asignación por hijo estipulada en el régimen contributivo de asignaciones familiares (Ministerio de Economía de la Nación [Mecon], 2009). Al igual que otras políticas similares, la AUH se centra en transferencias directas de dinero, focalizadas en los niños hasta los 18 años y transferibles a los mayores responsables de su guarda y cuidado.

En 2011, la AUH se complementa con la creación y articulación de la Asignación Universal por Embarazo (AUE), reconociendo de esta manera también el derecho de los niños en el vientre materno. De este modo se intenta paliar la mortalidad infantil a partir de la semana 12 del periodo de gestación, y para ello las gestadoras, madres beneficiadas, tienen que cumplir con todos los controles prenatales requeridos por el sistema público de salud, donde se instrumentalizan las condicionalidades.

Ahora bien, volviendo a la AUH, como derecho les corresponde a los hijos de las personas que están desocupadas, trabajan en la economía informal y demás situaciones que prescribe el programa. En este contexto, necesariamente se requiere de la acreditación anual de escolarización y controles de salud de los niños mediante la entrega de informes parciales mensuales; vale señalar que se abarca un rubro que va desde el nacimiento hasta los 18 años del menor, y un máximo de 5 hijos por familia, y se prioriza a los hijos discapacitados y a los de menor edad.

El estudio sobre este programa, realizado por Agis, Cañete y Panigo (2010), establece que "el análisis conjunto de resultados cualitativos y cuantitativos permite aseverar que la AUH es una medida de política social sumamente exitosa, especialmente en materia de su impacto sobre la indigencia y la desigualdad extrema entre ricos y pobres". (p. 11)

Sin embargo, el estudio que presentan Pautassi, Arcidiácono y Straschnoy (2013), que cuenta con un pormenorizado relevamiento de datos a través de entrevistas semiestructuradas a los beneficiarios, invita a reflexionar no sobre la eficacia del sistema o su potencialidad igualadora respecto a los beneficiarios que son los niños, sino, y fundamentalmente, frente a la posibilidad del rompimiento de situaciones de dependencia y dominación de la mujer que recibe los fondos. En este caso, su visión se centra ya no en el niño, cuyos beneficios hacia futuro son insoslayables en el marco de romper con la recreación de situaciones que permiten la traslación intergeneracional de la pobreza estructural. Esta cuestión que es absolutamente positiva, pese a la siguiente cita:

[El sistema] no rompe con la lógica dual en materia de política social ni con los sesgos de género que han caracterizado a las políticas sociales de la Argentina contemporánea, sino que sigue determinando el acceso a los satisfactores por la situación laboral de los adultos dejando a los niños, niñas y adolescentes vinculados directamente con tal situación. Una verdadera transformación, entre otras cosas, supone instalar esquemas de políticas sociales basados en principios de derechos que deslinden prestaciones de la condicionalidad laboral o de la condicionalidad reproductiva. Pero no sólo los niños y niñas quedan vulnerables a esta lógica sino las mujeres en edades centrales, al atar la percepción completa de la 
asignación al condicionamiento de la salud y educación, sin estimular políticas de formación o inserción laboral. (Pautassi, Arcidiácono y Straschnoy, 2013, p. 46)

Evidentemente, hay secuelas recientes tanto de la dictadura militar de 1976 como de 1983, así como del achicamiento del Estado neoliberal de los noventa con los procesos de privatización y flexibilización laboral, sus políticas restrictivas del gasto social y la destrucción de la industria nacional, lo cual terminó ahogando a la sociedad con una presión fiscal desmesurada. A ello se aúna el agotamiento del incesante endeudamiento con acreedores internacionales, que llevó al colapso y al corralito que se conoce como la gran crisis del 2001. Esta dinámica provocó de forma reaccionaria, luego de ese año crucial, un cambio paradigmático en el posicionamiento del Estado y respecto a su actuación. Por ello, con mayor intensidad desde el 2003 y hasta la fecha, pueden distinguirse tres tipos de políticas dirigidas a paliar la pobreza:

a) Políticas de transferencias directas - generalmente condicionadas- desde el ámbito nacional hacia las instancias subnacionales y locales.

b) Políticas de aumento de los ingresos mediante el perfeccionamiento del sistema tributario y la instauración de retenciones a las exportaciones de productos primarios, fundamentalmente.

c) Políticas de incremento de subsidios y ofertas de trabajo público o público-privado, y de esa manera el Estado asume el costo (total y parcial) de reestructuración no asumido por el mercado.

Siguiendo a Coraggio (2002) en la búsqueda de una economía justa, que solo puede ser adecuadamente juzgada en tanto y en cuanto contribuya al desarrollo de la sociedad en su conjunto, los Programas de Transferencias Monetarias Condicionadas (PTMC) se presentan a priori como nuevos instrumentos que coadyuvan a la receta keynesiana clásica para paliar los males de la economía recesiva, a través de una canalización de recursos públicos que se destinan deliberadamente a atacar la indigencia y pobreza, pero que al tiempo incentivan la actividad económica doméstica y el consumo. El informe Desarrollo social inclusivo de la Cepal (2015) establece en este sentido:

Lo social se ha fortalecido en la agenda pública y eso se ha expresado mediante nuevos compromisos jurídico-normativos y una diversidad de experiencias positivas en cuanto a capacidades fiscales, técnicas, organizacionales y de coordinación interinstitucional; por ello, se requiere profundizar dichos procesos, propiciando una mayor sostenibilidad en políticas orientadas por principios de integralidad, efectividad, eficiencia, participación y transparencia. (p. 144)

Por su parte, el Banco Mundial (2014) en su documento Conditional cash transfers reducing present and future poverty concluye:

La evaluación de la experiencia de las TMC, hasta ahora, confirma que los programas fueron eficaces en el sentido de que existen pruebas sólidas de su impacto positivo en la reducción de la pobreza a corto plazo y en el aumento del uso de servicios de educación y salud. Estos logros no deben minimizarse porque demuestran firmemente que los programas públicos bien diseñados pueden tener efectos significativos en indicadores sociales críticos. (p. 27)

A pesar de que la República Argentina cuenta con una gran extensión territorial $\left(2.800 .000 \mathrm{~km}^{2}\right.$, aproximadamente), constituye un rasgo característico del país, por motivos sociohistóricos, la concentración poblacional en un territorio reducido con grandes ciudades. Tal es así que el $66,7 \%$ de la población reside en las provincias de Buenos Aires (39\%), Córdoba (8,2\%), Santa Fe (8\%), Mendoza $(4,3 \%)$ y la Ciudad Autónoma de Buenos Aires (C.A.B.A.) (7,2\%). Consecuentemente, se originan 
centros urbanos con una densidad poblacional que sobrepasa ampliamente a otros. A modo de ejemplo, mientras que C.A.B.A. posee una densidad poblacional de $14.450,8$ habitantes por $\mathrm{km}^{2}$, existen provincias en las cuales hay menos de un habitante por $\mathrm{km}^{2}$.

Ahora bien, este análisis de relación vertical en la generación de políticas públicas de reducción de la pobreza y la indigencia, así como respecto a la consolidación de una clase media trabajadora, debe necesariamente contrastarse con la nivelación horizontal de aprovechamiento de oportunidades por región del país. Para ello se eligió, como dato ilustrativo, representar en la gráfica 1 los datos que ofrece el Indec (2010) frente a las necesidades básicas insatisfechas de los habitantes de Argentina, atendiendo a una división provincial. A partir del Censo Nacional de Hogares del 2010 se vislumbran las divergencias que presentan las distintas regiones.

Cómo indica esta gráfica, existen realidades absolutamente dispares entre las distintas provincias del país. Así, mientras el porcentaje de personas en situación de pobreza en Santa Cruz es del $1,1 \%$, en la provincia del Chaco es del $14,2 \%$. Por otro lado, y complementando este análisis, se muestran más de 13 puntos porcentuales de diferencia entre ambos extremos comparativos provinciales; así, en el análisis de la renta per cápita de los habitantes de cada provincia se ve particularmente vislumbrado el grado de contraste y desigualdad entre las regiones de Argentina.

Otro punto relevante de concentración y desigualdad se observa en la gráfica 2, que muestra la distribución porcentual del producto bruto geográfico nacional en 2009, por región. La limitante de adoptar este año y no uno más reciente está dada por la información que suministra el Ministerio de Gobierno y Reforma del Estado, Secretaría de Tecnología para la Gestión; sin embargo, es actual y representativa de lo que pretende establecerse en este escrito, puesto que se recoge información pública. Vale señalar que no se realiza un análisis empírico cuantitativo directo por cuanto ello escapa del objeto de esta investigación.

Porcentaje de población en Argentina con Necesidades Básicas Insatisfechas

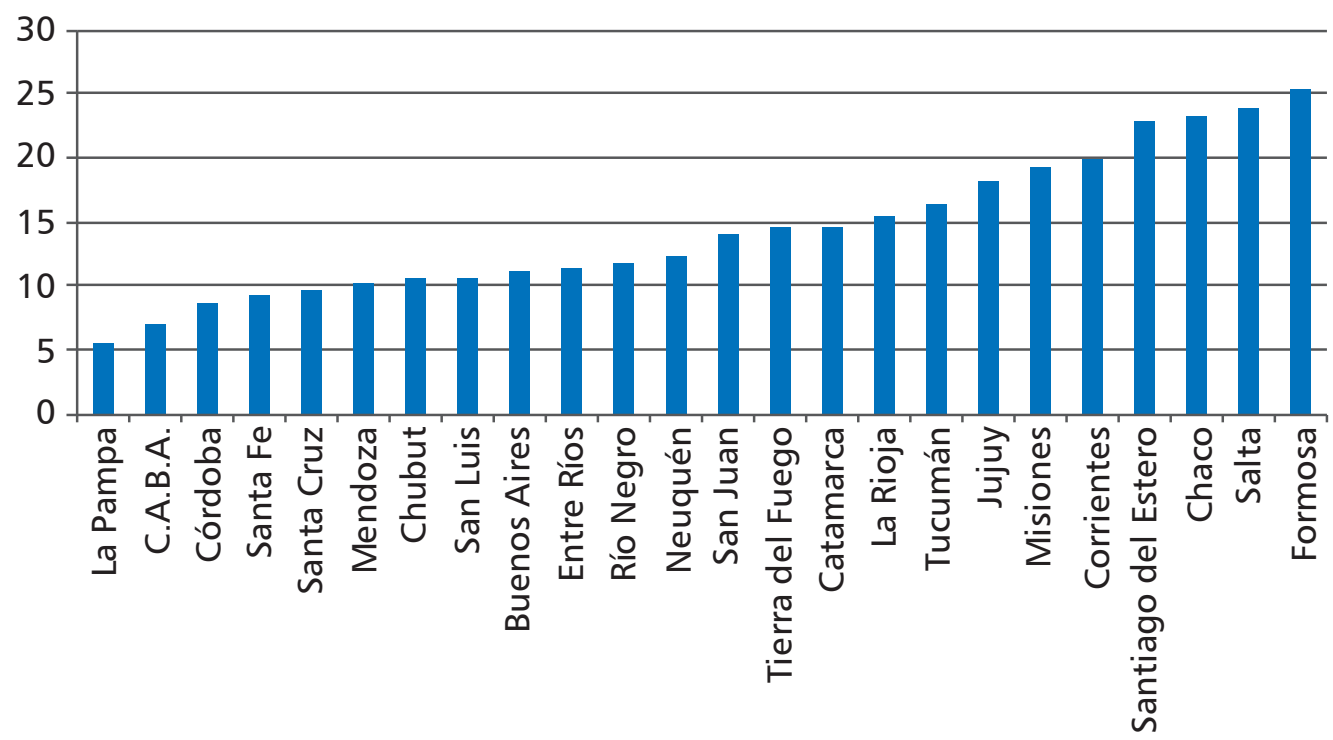

Fuente: Indec (2010). 
Distribución porcentual del producto bruto geográfico nacional en 2009, por región

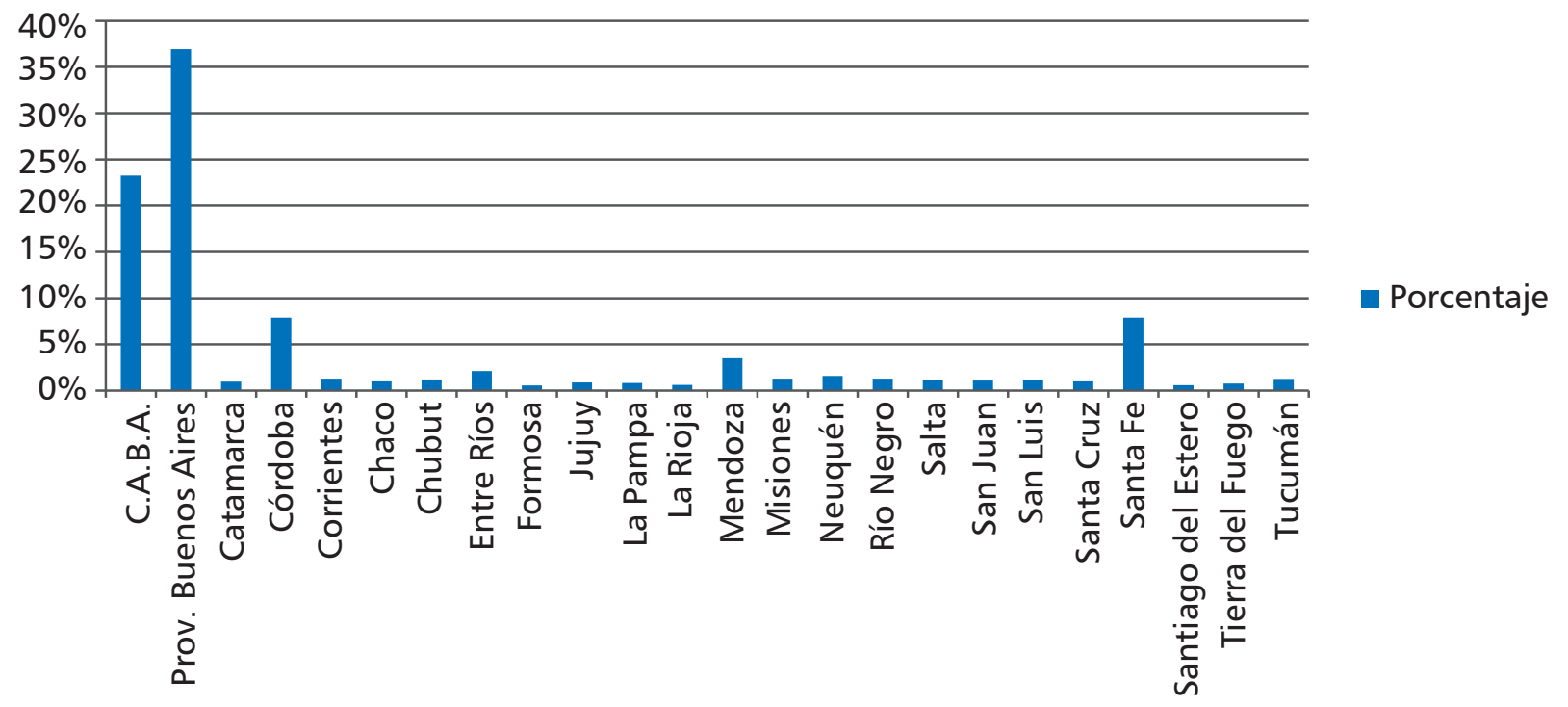

Fuente: Ministerio de Gobierno y Reformas del Estado, Secretaría de Tecnología para la Gestión (2009).

Vislumbrada la desproporcional generación de valor a lo largo y ancho del Estado argentino (Rezzoagli y Gamberg, 2015), puede observarse de forma aleatoria otra variable importante: el acceso a la educación, el cual representa uno de los indicadores que de manera más clara pone al desnudo la necesidad de profundizar este sendero en el que se ha comprometido el país durante los primeros 14 años poscrisis 2001, poniendo especial énfasis en los desequilibrios entre las regiones argentinas, de las más pobladas a las de media o baja densidad. En esta materia es importante destacar que mientras en la Ciudad Autónoma de Buenos Aires (C.A.B.A.) un $82 \%$ de la población asistió y completó el nivel primario, en tres provincias del norte argentino: Corrientes, Chaco y Misiones, los porcentajes descienden al $56,1 \%, 53,6 \%$ y $51 \%$, respectivamente (Indec, 2015).

Por otro lado, en cuanto a la asistencia a establecimientos educativos entre los 15 y 17 años de edad, C.A.B.A. vuelve a mostrar los niveles más altos: $90,5 \%$, solamente superada por Tierra del Fuego con el 94,8\%. En contraste, en Santiago del Estero y Misiones solo concurren el 66,5\% y
71,6\%, respectivamente (Indec, 2015). En este línea, y llevando la comparación a la totalidad de las provincias argentinas, en función de los datos reflejados por el Indec, los niveles de finalización del secundario son visiblemente dispares aunque sin pronunciamientos estrepitosos; esto último se debe principalmente, según se aprecia del análisis correlacionado, a la AUH.

No obstante el avance en la lucha contra la mendicidad, el trabajo infantil, la desnutrición, el cuidado a la salud, la educación y, en definitiva, el rompimiento de cadenas intergeneracionales de la pobreza estructural en ciertos sectores poblacionales, hay cuestiones que deben (re)discutirse en las TMC para romper el proceso desigualador horizontal, atendiendo a variables exógenas como el lugar de origen o la habitabilidad. El problema clave de la desigualdad de la educación, por ejemplo, reside también en la cantidad, pero sobre todo en la calidad. Si no hay recursos económicos suficientes para mejorar la cantidad y calidad del sistema público, que es el que puede cerrar brechas en personas de escasos recursos, el esfuerzo lamentablemente siempre será insuficiente. 
No basta con poner a los niños en el aula - situación que en sí misma es sumamente importantesino también asegurar un banco por cada niño, además de las condiciones humanas, técnicas e infraestructurales para una educación de calidad.

En Argentina, la AUH es un programa nacional, en tanto la responsabilidad del funcionamiento educativo y sanitario es exclusivamente provincial (subnacional). Y si bien la nación colabora con programas específicos, la educación primaria y secundaria está descentralizada a dichos gobiernos regionales, por lo que el financiamiento del sistema no va de la mano con el financiamiento del programa. En la realidad, la falta de articulación provoca déficit educativo y el fracaso del programa, y no porque no haya interés por parte de sus beneficiarios, sino por respuestas inadecuadas de los entes que prestan los servicios, que en muchas ocasiones, además, están muy lejos del lugar de habitabilidad de los beneficiarios del programa.

Existen otras variables que podrían considerarse para plantear las asimetrías regionales, fundamentalmente a escalas territoriales de segundo nivel o más específicas (Rezzoagli y Bazza, 2012), y respecto al empleo formal y la generación de producto bruto geográfico (Rezzoagli y Gamberg, 2014); sin embargo, para los fines de este artículo, se considera que se ha cumplido con el desafío de demostrar la existencia de desigualdades horizontales que implican un fortalecimiento de los planes y programas existentes, pero también la imperiosa necesidad de un replanteamiento de las relaciones escalares de gestión de estos.

Por lo analizado hasta aquí, pareciera que se está ante una realidad paradójica. Es indiscutible que en los últimos quince años poscrisis 2001 se ha dado el aumento y la consolidación de la clase media, así como el crecimiento significativo del nivel de instrucción y educativo, convalidado por los índices de matriculación y egresos. También se ha producido una reducción comprobada de los niveles de pobreza e indigencia en el país. Sin embargo, frente a estos importantes logros, puede vislumbrarse la dispar efectividad que tuvo en cada región del país individualmente considerada, lo cual lleva a pensar que dicha reducción estuvo focalizada en las regiones más pobladas y, por tanto, con mayor nivel de desarrollo (pampa húmeda).

Ahora bien, los desafíos y las problemáticas que presentan los programas justamente se manifiestan por falta de acuerdos o arreglos político-institucionales necesarios ${ }^{7}$. Por ejemplo, de nada sirve exigir la verificación de un calendario de vacunación para la entrega de dinero en efectivo a sus beneficiarios cualificados, en función de reducir la pobreza y las enfermedades, si cuando estos van al dispensario o al centro de salud se encuentran con que las vacunas no están disponibles o se han agotado. Lo mismo ocurre cuando los sistemas educativos son precarios y a duras penas cumplen con la función básica de formación, o si el hospital que atiende parturientas se encuentra a muchos kilómetros de distancia del domicilio de la beneficiaria.

Las TMC necesitan de un andamiaje estatal inteligente: otorgar las prestaciones a los sectores que realmente lo necesitan, evitando así lo que de otro modo sería un dispendio de recursos públicos en aras a un clientelismo político; de igual manera, garantizar la infraestructura necesaria para que los beneficiarios puedan cumplir con los hechos condicionantes que se les exigen de manera efectiva, y procurar que la medida en sí misma sea siempre temporal y encaminada como fin último a erradicar los focos de pobreza.

Este ensamblaje estatal necesario, que se entiende como el arreglo público que rodea a la prestación misma y da sustento material efectivo a las condicionalidades de esta, es un elemento esencial para el éxito de los programas y plantea la necesidad de discutir, con una visión más holística, desde dónde se adoptan las decisiones políticas y qué arreglos tienen entre los distintos entes gubernamentales que conforman los diversos estratos verticales del poder decisorio.

$7 \quad$ Para Scharpf (1997) hay que tener en cuenta la interacción de dos conceptos claves: actores e instituciones; así se puede diagnosticar, estudiar y prever el desarrollo de las políticas públicas. 


\section{EL FEDERALISMO FISCAL COMO FENÓMENO PROBLEMÁTICO}

Es importante manifestar que en una Argentina asimétrica, el federalismo fiscal como fenómeno e instrumento igualador primordialmente debe centrar su análisis en el mejoramiento de la vinculación horizontal de las instancias subnacionales entre sí, para luego, y en función de lo anterior, evaluar la adecuada o inadecuada vinculación vertical entre el Gobierno Nacional y las instancias subnacionales (Rezzoagli y Bazza, 2012). Cetrángolo (2005) afirma en este sentido:

En la Argentina [...] la discusión ha estado casi exclusivamente centrada en la posibilidad de sancionar un nuevo régimen de reparto de impuestos y poco se ha avanzado en el debate de un federalismo fiscal más maduro que facilite una mejor calidad de vida a lo largo de su territorio, [...] una Asamblea Constituyente estableció la necesidad de un nuevo régimen que nunca pudo ser acordado. Los pactos transitorios, acuerdos bilaterales (muchas veces secretos) y la desconfianza mutua han reemplazado a las reglas transparentes y estables, la confianza y la complementariedad que deben dominar un sistema federal moderno que priorice el consenso por sobre el conflicto. (p. 34)

La Constitución de la Nación Argentina en su artículo 75, inciso 2, se refiere a las potestades tributarias que les corresponden a los ámbitos nacional y subnacional. Los incisos 12, 13 y 18 de este artículo se refieren, respectivamente, al dictado de la legislación de fondo, a la facultad de reglar el comercio internacional e interprovincial y a la llamada "cláusula del progreso" por la cual el Congreso Nacional ha podido establecer exenciones de tributos locales. De igual manera, el inciso 19 hace referencia expresa a la aceptación de la existencia de profundas asimetrías territoriales y a la necesidad primordial del Estado de actuar para combatirla.
En 1988 el Congreso sancionó la última y vigente Ley 23.548, sobre coparticipación federal de impuestos. A través de esta, entre otras disposiciones, se amplía la masa coparticipable, al incluirse todos los impuestos nacionales existentes o por crearse (artículo 2), y se mejora la participación provincial en la distribución primaria (artículo 3). Asimismo, el artículo 3 dispone que la distribución primaria se efectúa en los siguientes porcentajes: nación, 42,34\%; provincias, 54,66\%; fondo de aportes del tesoro nacional a las provincias, $1 \%$; recupero del nivel relativo de ciertas provincias, $2 \%$.

En 1992 y 1993 se firmaron entre las autoridades del Gobierno de nación y los gobernadores de las provincias los Pactos Fiscales I y II, de marcada tendencia neoliberal, y signados por una impronta ideológica de retroceso en la intervención del Estado en la economía. Brindaban una cantidad de recursos coparticipables a cada provincia, con el requerimiento de favorecer las inversiones internacionales en sus territorios, descentralizar funciones y gastos públicos indispensables que eran desarrollados por el Estado Nacional hacia estas, auspiciar políticas legislativas provinciales que garantizaran el perfeccionamiento del mercado otorgando exenciones impositivas a capitales foráneos, privatizar total o parcialmente las empresas públicas y dar concesiones totales o parciales de servicios públicos, de prestaciones $\mathrm{u}$ obras a empresas privadas, con lo cual se favorecía el alejamiento del Estado y se auspiciaba la desregulación y flexibilización laboral.

Además, las provincias se obligaban a derogar ciertos impuestos, como aquellos que gravaban los intereses de depósitos a plazo fijo y en caja de ahorro, los aplicados o los débitos bancarios, entre otros; también debían modificar el impuesto sobre los ingresos brutos y los impuestos sobre la propiedad inmobiliaria, con un claro tinte de favorecer al mercado y los capitales especulativos, aun contra el propio desfinanciamiento de las arcas públicas. Asimismo, se obligaban a un cambio en el sistema previsional, que pasaba de ser público a público-privado a través de las administradoras de fondos de jubilaciones y pensiones (AFJP). Por su 
parte, el Gobierno Nacional aumentaba el piso de distribución a las provincias como contrapartida.

En 1994 llega la reforma del texto constitucional argentino, en sintonía con este claro posicionamiento estatal de dejar el dinero en manos de los particulares como motor del desarrollo, con el achicamiento del Estado y el vaciamiento infraestructural y humano de sus capacidades endógenas de actuación.

La receptada solidaridad fiscal incorporada en dicha reforma persuade acerca de la función redistributiva que se le atribuye al régimen de coparticipación federal de impuestos. En ese orden de ideas, indican Rezzoagli y Bazza (2012):

Cuando el texto constitucional señala criterios objetivos para la coparticipación, pero exige además, explícitamente, su carácter equitativo y solidario para dar prioridad al logro de un grado equivalente de desarrollo, calidad de vida e igualdad de oportunidades en todo el territorio nacional. (p. 66)

El artículo 75, inciso 19, permite visualizar un reforzamiento de lo anteriormente analizado, pues establece la obligación del Estado de proveer políticas públicas diferenciadas, atendiendo para ello al dispar grado de desarrollo de las provincias y regiones del país. Así pues, expresamente se reconoce la importancia del principio de solidaridad como premisa rectora en la forma de articular la distribución de los recursos fiscales junto con parámetros de efectividad; un sistema justo que se base exclusivamente en cuestiones de eficiencia o correspondencia fiscal es injusto y generador de brechas cada vez más amplias (Llach, 2013, pp. 30-31).

Así, luego de la reforma constitucional donde se establece que las dos distribuciones de recursos (primaria y secundaria) deben hacerse de acuerdo con la solidaridad, impone que tanto el Gobierno federal como las provincias más desarrolladas se comprometan con el destino de las más desfavorecidas (Corti, 2007).
Pese a las buenas intenciones de la reforma constitucional, y aun cuando se encuentra ampliamente vencido el plazo para sancionar el nuevo régimen de coparticipación federal de impuestos (que exigía la cláusula transitoria sexta de dicha reforma de 1994), actualmente se encuentra postergado el dictado de la nueva ley-convenio que responda a criterios de equidad, solidaridad y redistribución; de hecho, se mantiene un sistema de reparto desigual y discrecional. Otra evidencia del problema es el reparto del distorsivo impuesto a los débitos y créditos bancarios, instaurado en el 2001 y vigente durante años gracias a las sucesivas prórrogas.

Con la sanción de la Ley 25.570 el 3/5/02, el Gobierno Nacional ratifica el "Acuerdo NaciónProvincias sobre Relación Financiera y Bases de un Régimen de Coparticipación de Impuestos", que fue celebrado el $27 / 2 / 02$. En ese momento poscrisis 2001, esto les permitió a las provincias mejorar su posición fiscal relativa, por cuanto pudieron retornar al régimen anterior al compromiso federal de $1999^{8}$ de distribuciones automáticas y por coeficiente, y optar por el menor pago de servicios producto del canje de la deuda y del límite del 15\% en la afectación de coparticipación para el pago de servicios de deuda.

La estructura tributaria argentina no es progresiva, sino todo lo contrario, y es dable destacar que no ha habido ningún intento de modificar esta situación estructural en casi un siglo. En el plano ideal, el sistema tributario argentino debe ser un instrumento igualador de capacidades económicas, que fortalezca y fomente el desarrollo a los de más bajos recursos y aplique mayor presión tributaria a los de altos recursos, con el objetivo de establecer una clase media estructural y un adecuado funcionamiento recursivo del Estado en función de estos parámetros. Sin embargo, el sistema tributario es nulo, no hay progresividad; el sistema fiscal favorece

$8 \quad$ Ley 25.235 sancionada el 15/12/99 y publicada en el Boletín Oficial el 7/1/00, que ratificó el acuerdo suscrito entre el Gobierno Nacional y los gobernadores electos de las provincias, denominado "Compromiso Federal", que fue llevado a cabo el 6/12/99. 
cada vez más la separación de clases y el empobrecimiento de los que menos tienen, pues la presión tributaria ejercida a estos sectores es más intensa, cuando un efectivo y correcto diagrama fiscal debería propender absolutamente a lo contrario.

Así, las reformas y medidas adoptadas desde el ámbito detractivo tuvieron efectos regresivos; esto es, utilizando las palabras de Num (2011), "Ios impuestos generan desigualdad". Aunado a esto, Gatti y Puig (2011) sostienen:

La fuerte disociación entre las responsabilidades de gastar y de recaudar, junto con un pobre diseño de los sistemas de transferencias establecidos por la Ley de Coparticipación federal vigente, constituyó un incentivo para la indisciplina fiscal y el endeudamiento excesivo, convirtiéndose así en el primer gran obstáculo para la implementación de una política fiscal contracíclica. (p. 3).

En el plano estrictamente recaudatorio, debe hacerse mención y reiterar la importancia de una presión tributaria progresiva como parámetro igualador que no existe en este país. Si se visualizan los impuestos vigentes con una supuesta progresividad en Argentina, aunque las alícuotas aumentan en relación con el incremento de la capacidad económica de los sujetos captados en los impuestos respectivos, la presión tributaria no lo hace en igual proporción, sino que disminuye. Un ejemplo de ello - aunque es una cuestión reiterada en todos- se encuentra en el impuesto a los bienes personales, que inicia con una alícuota del $0,50 \%$ aplicada a la base imponible cuando el valor total de los bienes gravados sea mayor a $\$ 305.000$ (mínimo no imponible) y hasta $\$ 750.000$ (primera escala), y culmina (última escala) con una alícuota del $1,25 \%$ cuando el valor total de los bienes gravados sea mayor a $\$ 5.000 .000$.

Así, puede observarse que la capacidad económica gravada aumenta más de $1500 \%$ y, sin embargo, la alícuota incrementa su presión tributaria en menos de un $300 \%$, y es mucho peor si se considera que la ley establece que sea esa alícuota del 1,25\% para un valor total de los bienes sujetos al impuesto mayor a $\$ 5.000 .000$; por ello, si el valor es de $\$ 15.000 .000$ o de $\$ 100.000 .000$, la alícuota sigue siendo la misma, no importa en cuánto aumente la capacidad económica del sujeto en función de los bienes acumulados. Esto presenta un claro ejemplo de regresividad, al disminuir abruptamente la presión tributaria en función del aumento de la capacidad económica, aun cuando las alícuotas aumenten, pues no sigue la equivalencia correspondiente.

Lamentablemente, este no es un caso aislado, pues dicha situación se repite en todos los impuestos graduados con una supuesta progresividad de alícuotas, aunque con un marcado descenso de la presión tributaria a medida que aumenta la riqueza. De este modo, se visualiza uno de los principales problemas del sistema detractivo argentino.

Aunado a lo anterior, puede reflexionarse sobre los dos más importantes tributos de Argentina en función de su porcentaje de recaudación y evasión. El impuesto a las ganancias (sociedades y personas físicas) tiene un amplio porcentaje de evasión fiscal y su recaudación en términos de PBI sigue siendo muy baja en comparación con países centrales. Si se le añade la elusión fiscal y la falta de intención política real en una recaudación progresiva, la conclusión es que gran parte de las posibilidades recaudativas de este impuesto no se cumplen. Indudablemente, una de las causas dependientes más importante la constituyen las numerosas exenciones y beneficios fiscales que, bajo ropaje de progreso, no hacen más que beneficiar a las rentas del capital y a los grupos de presión, tales como las que se generan por la compraventa de acciones, por los dividendos, por las transacciones financieras, por los intereses de los títulos públicos, entre otras.

Respecto del IVA, se coincide con Num (2016) cuando establece:

El total de los gravámenes al consumo duplica lo que se recauda por ganancias, y sitúa a la Argentina por encima del promedio tanto de América Latina como de 
los países de la OCDE. [...] La evasión es elevada y difícil de combatir. Si la evasión descendiera a los niveles que alcanza en Chile, por ejemplo, la tasa general del $21 \%$ podría rebajarse entre 6 y 8 puntos porcentuales. (p. 1)

La redistribución del ingreso en Argentina, sin lugar a dudas, es progresiva en función de lo analizado en el epígrafe anterior, fundamentalmente debido a los programas y las transferencias de corte social, aun con las dispares oportunidades de aprovechamiento que tuvo en regiones particularmente visualizadas.

Existen dos grandes desafíos en Argentina que atañen a un conjunto de problemáticas conexas e implícitas que se han de solucionar. Por un lado, en materia de recaudación tributaria, está la necesidad de fomentar la equidad a través de una reforma impositiva verdaderamente progresiva, en atención a cada impuesto en particular, hasta la instauración de un sistema que en su conjunto favorezca dicho objetivo. En segundo lugar, lograr que el sistema financiero, si bien progresivo, medido hasta el 2015, sea efectivo en atención a las necesidades de cada región de forma diferencial y solidaria, atendiendo a un proceso bottom-up para el aprovechamiento de oportunidades.

\section{NOTAS FINALES}

Por lo expuesto anteriormente, puede observarse que el Gobierno Nacional ha disminuido históricos gastos y servicios a su cargo en las últimas décadas como resultado de un proceso de descentralización fundamentalmente hacia las provincias, pero que, pese a esto, continúa detentando recursivamente la masa principal de los ingresos fiscales. La consecuencia de ello es la profundización de un desequilibro fiscal vertical que llama a la reflexión, especialmente vinculado a la falta de coordinación efectiva entre las actuaciones centrales y subnacionales tendientes a lograr mayor efectividad de los programas diagramados e instrumentalizados, con recursos nacionales, desde el gobierno central a todos los rincones del país, a través de las oficinas de la Administración Nacional de la Seguridad Social (Anses), entes descentralizados, o también a través de los municipios y distintos órganos provinciales. Esto, en ocasiones, produce en desmedro de las posibilidades de los gobiernos subnacionales para brindar recursivamente las condiciones técnicas, humanas y estructurales colindantes que permitan mayor eficiencia en los resultados esperados por dichos programas; en suma, se generan desequilibrios en el aprovechamiento de oportunidades para los ciudadanos.

Así pues, aunque se reduce la corresponsabilidad fiscal y se profundiza la ilusión fiscal en las distintas regiones del país, estas cuestiones pueden ser salvables con la incorporación de paulatinos procesos de efectividad recursiva y corresponsabilidad, a medida que se avance en los objetivos de nivelación y equidad horizontal. Lo que resulta preocupante es que esto último tampoco se logre de forma proporcional en todos los ámbitos territoriales y poblacionales; por ello, aunado a las consecuencias fiscales negativas, la dispar efectividad de estos programas centrales favorece la efectiva reducción de la pobreza y de las necesidades básicas insatisfechas exclusivamente en las zonas centrales, pero cualifica y profundiza la pobreza y las carencias para la consecución de una vida digna en zonas periféricas de Argentina. La situación es contradictoria: en términos de promedios nacionales es indiscutible el avance logrado, pero junto a esto se vislumbra centralización de los efectos positivos en las zonas más desarrolladas y pobladas, mientras que se acrecienta la brecha con las provincias restantes, las cuales terminan "exportando" sus habitantes a las ciudades y regiones en mención, debido a la búsqueda de mejores condiciones de vida.

¿Cómo propender a la igualación de oportunidades desde la estructura financiera y tributaria en un país asimétrico que pertenece a un continente profundamente desigual? No debe soslayarse la importancia que tiene un adecuado 
perfeccionamiento del sistema detractivo o tributario, que atienda a una efectiva progresividad, gravando con mayor presión tributaria las capacidades económicas más elevadas; cuestión que en la actualidad no sucede, en la medida en que se visualiza un sistema impositivo con marcado tinte regresivo, lo cual atenta contra el principio de igualdad, la consolidación de una clase media argentina y la disminución de la brecha entre ricos y pobres.

Este es un desafío principal en el que la medición de las necesidades básicas satisfechas y la capacidad económica deben tener una vinculación mucho más estrecha con el diagrama de la presión fiscal, determinada en función de los montos imponibles y las alícuotas instrumentalizadas al efecto, además de repensar la instrumentalización y efectividad del gasto tributario (entendido como la exorbitancia de incentivos fiscales actualmente en vigencia).

Desde la órbita del sistema presupuestario y financiero, existen aspectos negativos en la distribución solidaria y las políticas de nivelación horizontal, como justamente la falta de correspondencia entre lo que se aporta y se obtiene, el clientelismo que favorece la relación política subordinada antes que la eficiencia económica y administrativa, la ilusión fiscal entre lo que se recauda y lo que se gasta y la falta de certeza en el mantenimiento prolongado de la bonanza de recursos, por carecer de un sistema de acumulación legítimo y efectivo. Sin embargo, estos problemas constituyen un mal menor inicial frente a la posibilidad igualadora efectiva y bien estructurada para la reducción de dichas asimetrías. Justamente, un sistema redistributivo bien encauzado y consensuado de abajo hacia arriba, aunque también necesariamente coordinado desde el ámbito central, tiende con el tiempo a superar dichos aspectos negativos, mediante paulatinos procesos de incorporación competencial y de correspondencia fiscal.

Evidentemente, esta injerencia del Estado en pos de reducir los márgenes de pobreza e indigencia ha dado resultados positivos y se ha traducido en una reducción de estos flagelos en los promedios nacionales. No obstante, volviendo a la consideración central de este artículo, debe necesariamente replantearse el modo de efectivización de dichos planes o programas, que son proclives a consolidar y aumentar el acaparamiento de oportunidades de las regiones más pobladas o con mayores estructuras y capacidades endógenas de actuación.

Un ejemplo ilustrativo es el de la provincia de Formosa, que según los datos del Indec (2010) constituye la región con mayor índice de hacinamiento de habitantes por vivienda; sin embargo, no es la provincia prioritaria para los créditos hipotecarios impulsados por el Gobierno Nacional (Plan Procrear $\left.{ }^{9}\right)$, pues el mayor número de beneficiarios se encuentra en la zona centro (provincias pujantes y con menor índice porcentual de hacinamiento). De acuerdo con los registros oficiales de la Anses, la distribución geográfica de dichos créditos fue la siguiente: casi un $70 \%$ se otorgó para la zona centro, un $9 \%$ se destinó a la Patagonia, otro $9 \%$ se distribuyó en Cuyo, un $8 \%$ se giró al noroeste argentino y un $5 \%$ se concedió al nordeste argentino.

La vinculación de los índices de pobreza, las necesidades básicas insatisfechas y las deficiencias regionales estructurales debe ser el motor para el cumplimiento de los incisos 2 y 19 del artículo 75 constitucional, para así perfeccionar la solidaridad y las políticas públicas financieras diferenciadas en un ámbito de consenso intergubernamental, como premisa para el desarrollo social y el cumplimiento efectivo de los derechos humanos para todos los habitantes del país, pues la nivelación horizontal es el principal escalón para cualquier tipo de mejoramiento estructural, técnico, recursivo y humano.

\section{REFERENCIAS}

9 El Programa Crédito Argentino del Bicentenario para la Vivienda Única Familiar (Pro.Cre.Ar), creado por Decreto 902/2012 del Poder Ejecutivo Nacional, ha sido hasta 2015 uno de los programas sociales con mayor derrame positivo en la sociedad, por cuanto permitió el acceso a vivienda propia en fracciones poblacionales de bajos y medios recursos que tenían vedada la posibilidad real de acceder a créditos bancarios para construcción de vivienda, además de la cantidad importante de puestos de trabajo directos e indirectos que promovió y el consumo de bienes ligados a la construcción. 
1. Agis, E., Cañete, C. y Panigo, D. (2010). El impacto de la Asignación Universal por Hijo en Argentina. Recuperado de http://www.trabajo.gov.ar/left/estadisticas/documentossubweb/area1/documentos/ auh_en_argentina.pdf

2. Anderson, E. (1999). What is the point of equality. Ethics, 109(2), 287-337.

3. Argañaraz, N., Barraudy, A. y Bulgarelli, V. (2013). Coparticipación federal de impuestos durante el año 2012 (Informe Económico, 203). Córdoba: Instituto Argentino de Análisis Fiscal.

4. Banco Mundial (2014). Conditional cash transfers reducing present and future poverty. Recuperado de https:// openknowledge.worldbank.org/handle/10986/2597

5. Banco Mundial (2015). Informe sobre el desarrollo. Recuperado de http://www.worldbank.org/en/publication/ wdr2015

6. Barbieri, T. (2007). Equidad de género, políticas y plan de acción. Buenos Aires: Ministerio de Trabajo, Empleo y Seguridad Social.

7. Cao, H. y Vaca, J. (2006). Desarrollo regional en la Argentina: la centenaria vigencia de un patrón de asimetría territorial. Revista Eure, 32 (95), 95-111.

8. Cecchini, S. y Vargas, L. H. (2014). Los desafíos de las nuevas políticas de desarrollo social en América Latina. Revista de Economía Crítica, 18, 113-135.

9. Comisión Económica para América Latina y el Caribe (Cepal) (2013). Lineamientos estratégicos para el desarrollo industrial en Argentina. Recuperado de http://archivo.cepal.org/pdfs/2013/S2013348.pdf

10. Comisión Económica para América Latina y el Caribe (Cepal) (2015). Desarrollo Social Inclusivo. Una nueva generación de políticas para superar la pobreza y reducir la desigualdad en América Latina y el Caribe. Recuperado de www.cepal.org/es/publicaciones/39100-desarrollo-social-inclusivo-nueva-generacion

11. Cohen, G. A. (1991). Incentives, inequality, and community. Recuperado de http://tannerlectures.utah. edu/_documents/a-to-z/c/cohen92.pdf

12. Cetrángolo, O. (2005). Coparticipación: revisión de los consensos. En La coparticipación y su laberinto: Ponencias del VIII Seminario sobre Federalismo Fiscal (pp. 33-37). Recuperado de www.cippec.org/ documents/10179/58583/L+Fiscal,\%20Coparticapaci\%C3\%B3n+en + su +laberinto,\%202006.pdf/ f5a4d1c6-45be-4f26-b1fa-2deb8c8b62c7

13. Cogliandro, G. (2010). El programa Asignación Universal por Hijo y los cambios en los programas de transferencias condicionadas. Recuperado de https://mpra.ub.uni-muenchen.de/23846/1/MPRA_paper_23846.pdf

14. Coraggio, J. L. (2002). La economía social como vía para otro desarrollo social. Recuperado de http://www. top.org.ar/ecgp/FullText/000000/CORAGGIO\%20Jose\%20Luis\%20-\%20la\%20economia\%20social.pdf

15. Corti, H. (2007). Derecho constitucional presupuestario. Buenos Aires: Lexis Nexis.

16. Etchichury, H. (2013). Igualdad desatada. La exigibilidad de los derechos sociales en la Constitución argentina. Córdoba: Universidad Nacional de Córdoba.

17. Fernandez, V., Seval, M. y Vigil, J. (2013). Institucionalidad regional en la Argentina reciente: el dilema de la política pública de desarrollo regional. Revista Brasileira Desenvolvimento Regional, 1(2), 77-105.

18. Fondo de las Naciones Unidas para la Infancia (Unicef) (2016). Bienestar y pobreza en niñas, niños y adolescentes en la Argentina. Recuperado de www.unicef.org/argentina/spanish/monitoreo_Pobreza_Completo.pdf

19. Fondo Monetario Internacional (2014). De la estabilización a un crecimiento sostenido. Recuperado de http:// www.imf.org/external/spanish/pubs/ft/ar/2014/pdf/ar14_esl.pdf 
20. Gatti, N. y Puig, J. (2011). Prociclidad del gasto público en Argentina e implicancias para el diseño de políticas de federalismo fiscal. Recuperado de http://www.researchgate.net/publication/260205438_

21. Hernández, A. M. (2010). La coparticipación impositiva federal. Buenos Aires: La Ley.

22. Instituto Nacional de Estadísticas y Censos (Indec) (2010). Censo Nacional de Hogares 2010. Recuperado de http://www.indec.gov.ar/nivel4_default.asp?id_tema_1=2\&id_tema_2 $=41$ \&id_tema_3 $=135$

23. Instituto Nacional de Estadísticas y Censos (Indec) (2010). Nivel de Educación Alcanzado 2010. Recuperado de http://www.indec.gob.ar/nivel4_default.asp?id_tema_1=4\&id_tema_2=33\&id_tema_3=97

24. Llach, J. J. (2013). Federales y unitarios en el siglo XXI. Buenos Aires: Temas.

25. Manos Unidas (2013). El desafío del hambre: la seguridad alimentaria en nuestro mundo globalizado. Madrid: Autor.

26. Ministerio de Gobierno y Reformas del Estado, Secretaría de Tecnología para la Gestión (2009). PBG: Producto Bruto Geográfico de las provincias argentinas. Recuperado de https://www.santafe.gov.ar/index. php/web/content/download/78880/381193/file/informe_PBG_oct_09.pdf

27. Narododowski, P. y Panigo, D. (2010). El nuevo modelo de desarrollo nacional y su impacto en la provincia de Buenos Aires. Cuadernos de Economía, 75. Recuperado de http://www.ec.gba.gov.ar/areas/Hacienda/ estudios_fiscales/UIM/Archivos/Cuaderno75.pdf

28. Novick, S. (2010). Políticas migratorias en la Argentina: experiencias del pasado, reformas actuales y expectativas futuras. En Estado actual y perspectivas de las políticas migratorias en el Mercosur (pp. 25-52). Montevideo: Flacso/Unesco.

29. Nun, J. (2011). La desigualdad y los impuestos. Introducción para no especialistas. Buenos Aires: Claves para Todos.

30. Num, J. (2016). Entrevista periodística en relación al libro La desigualdad y los impuestos. Introducción para no especialistas. Recuperado de http://vocesenelfenix.com/content/acerca-de-la-desigualdad-y-los-impuestos

31. Observatorio de la Deuda Social Argentina (2016). Pobreza y Desigualdad por Ingresos en la Argentina Urbana 2010-2015. Tiempo de Balance. Buenos Aires: Universidad Católica Argentina.

32. Organización de Naciones Unidas (ONU) (2010). Informe sobre Desarrollo Humano. Recuperado de http:// hdr.undp.org/sites/default/files/hdr_2010_es_complete_reprint.pdf

33. Organización de Naciones Unidas (ONU) (2015). Desarrollo. Recuperado de http://www.un.org/es/ sections/what-we-do/promote-sustainable-development/

34. Oszlak, O. (2010). Formación histórica del Estado en América Latina. Elementos teórico-metodológicos para su estudio. En C. Acuña (Ed.), Lecturas sobre el Estado y las políticas públicas: Retomando el debate de ayer, para fortalecer el actual (1. a ed.) (pp. 115-142). Buenos Aires: Brujas.

35. Pautassi, L., Arcidiácono, P. y Straschnoy, M. (2013). Asignación Universal por Hijo para la Protección Social en Argentina. Santiago de Chile: Cepal. Recuperado de http://repositorio.cepal.org/bitstream/ handle/11362/6193/LCL3662_es.pdf?sequence $=1$

36. Piffano, H. (1998). Federalismo fiscal en Argentina. Ideas y propuestas sobre el nuevo acuerdo fiscal federal. Recuperado de http://faculty.udesa.edu.ar/tommasi/cedi/dts/dt2.pdf

37. Porto, A., y Sanguinetti P. (1993). Descentralización fiscal en América Latina: el caso Argentino. Santiago de Chile: Cepal.

38. Porto, A. (2003). Etapas de la coparticipación federal de impuestos. La Plata: Universidad Nacional de la Plata. 
39. Porto, A. (2004). Disparidades regionales y federalismo fiscal. La Plata: Universidad Nacional de la Plata.

40. Porto, A. (2008). El funcionamiento del federalismo fiscal. La Plata: Universidad Nacional de la Plata.

41. Programa de las Naciones Unidas para el Desarrollo (PNUD) (2010). Informe Regional sobre Desarrollo Humano para América Latina y el Caribe. Recuperado de http://hdr.undp.org/sites/default/files/rhdr2010-rblac.pdf

42. Rezzoagli, L. (2011). Asimetrías regionales y federalismo fiscal. Un enfoque jurídico-financiero-institucional del caso Argentino. DAAPGE, 15, 113-131.

43. Rezzoagli, L. y Bazza, A. (2012). Aspectos clave del federalismo fiscal y las asimetrías regionales en la República Argentina. Vía Iuris, 13, 61-72.

44. Rezzoagli, L. y Gamberg, G. (2014). The equality of opportunity: critical guidelines for the continuity of the argentine centre-periphery with continental contributions unequalizing process. Ecorfan Journal, 5(13), 2154-2177.

45. Rezzoagli, L. y Gamberg, G. (2015). El ciclo de dependencia centro-periferia en Argentina: revisión del producto bruto geográfico, el empleo formal y las políticas fiscales entre 1990 y 2010. Finanzas y Política Económica, 7(2), 257-278.

46. Roemer, J. (1998). Igualdad de oportunidades. Isegoría, 18, 71-87.

47. Scharpf, F. (1997). Games real actors play: Actor-centred institutionalism in policy research. Recuperado de https://westviewpress.com/books/games-real-actors-play/

48. Sen, A. (2000). Desarrollo y libertad. Recuperado de http://www.palermo.edu/Archivos_content/2015/ derecho/pobreza_multidimensional/bibliografia/Sesion1_doc1.pdf

49. Stumbro, G. y Rivas, D. (2013). La industria argentina frente a los nuevos desafíos y oportunidades del siglo XXI. Santiago de Chile: Cepal.

50. Tiebout, C. (1956). A pure theory of local expediture. The Journal of Political Economy, 64(5), 416-424.

51. Treber, S. (2004). Naturaleza y efectos de los impuestos distorsivos. XXXVII Jornadas Internacionales de Finanzas Públicas. Córdoba: Universidad Nacional de Córdoba.

52. Velázquez, G. (2008a). Las regionalizaciones argentinas: evolución de su capacidad de discriminación del bienestar de la población (1943-1992). GeoFocus, 8, 18-43.

53. Velázquez, G. (2008b). Geografía y bienestar. Situación local, regional y global de la Argentina luego del censo de 2001. Editorial Eudeba: Buenos Aires.

54. Velázquez, G. A. (2010). Geografía y bienestar en la Argentina: desigualdad de la sociedad y el territorio a comienzos del XXI. Geograficando, 6, 15-36.

55. Velázquez, G., Mikkelsen, C., Linares, S. y Celemín, J. P. (2014). Calidad de vida en Argentina: Ranking del bienestar por departamentos (2010). Tandil: Universidad del Centro de la Provincia de Buenos Aires. 